\title{
Neuronal Nuclear Organization Is Controlled by Cyclin-Dependent Kinase 5 Phosphorylation of Ras Guanine Nucleotide Releasing Factor-1
}

\author{
Sashi Kesavapany ${ }^{a}$ Tej Kumar Pareek ${ }^{b} \quad$ Ya-Li Zheng ${ }^{a} \quad$ Niranjana Amin ${ }^{a}$ \\ J. Silvio Gutkind ${ }^{c}$ Wu Ma ${ }^{d}$ Ashok B. Kulkarni ${ }^{b}$ Philip Grant ${ }^{a}$ Harish C. Pant ${ }^{a}$ \\ ${ }^{a}$ Cytoskeletal Protein Regulation Section, National Institute of Neurological Disorders and Stroke, \\ ${ }^{b}$ Functional Genomics Section, Craniofacial Developmental Biology and Regeneration Branch, and \\ 'Laboratory of Oral and Pharyngeal Cancer, National Institute of Dental and Craniofacial Research, \\ National Institutes of Health, Bethesda, Md., dCenter for Bio/Molecular Science and Engineering, \\ Naval Research Laboratory, Washington, D.C., USA
}

\section{Key Words}

RasGRF1 $\cdot$ p35 $\cdot$ Cdk5 $\cdot$ Ras $\cdot$ Phosphorylation $\cdot$ Nuclear organization

\begin{abstract}
RasGRF1 is a member of the Ras guanine nucleotide exchange factor (RasGEF) family of proteins which are directly responsible for the activation of Ras and Rac GTPases. Originally identified as a phosphoprotein, RasGRF1 has been shown to be phosphorylated by protein kinase $A$ and more recently, by the non-receptor tyrosine kinases Ack1 and Src. In this report we show that RasGRF1 interacts with and is phosphorylated by Cdk5 on serine 731 to regulate its steady state levels in mammalian cells as well as in neurons. Phosphorylation on this site by Cdk5 leads to RasGRF1 degradation through a calpain-dependent mechanism. Additionally, cortical neurons from Cdk5 knockout mice have higher levels of RasGRF1 which are reduced when wild-type Cdk5 is transfected into these neurons. In mitotic cells, nuclei become disorganized when RasGRF1 is overexpressed and this is rescued when RasGRF1 is co-expressed with active Cdk5. When RasGRF1 levels are elevated in neurons through overexpression of either the wild-type RasGRF1, or the phosphorylation mutant of RasGRF1 and by the transfection of a
\end{abstract}

dominant negative Cdk5 construct, nuclei appeared condensed and fragmented. On the other hand, a reduction of RasGRF1 levels through p35/Cdk5 overexpression also leads to nuclear condensation in neurons. These data show that phosphorylation of RasGRF1 by Cdk5 tightly regulates its levels, which is essential for proper cellular organization.

Copyright $\odot 2006$ S. Karger AG, Basel

\section{Introduction}

Cyclin-dependent kinase $5(\mathrm{Cdk} 5)$ is a member of the proline-directed serine/threonine kinases that is activated when associated with its activators p35 and p39 [1,2]. Cdk5 activity is critical in the proper formation of cortical structures, neurite outgrowth, neuronal migration during development and axon patterning [3,4]. It is also implicated in the regulation of cellular adhesion through its association with $\beta$-catenin $[5,6]$, synaptic vesicle release activity through its interaction with synapsin and Munc-18, endocytosis [7-12] and regulation of dopamine signaling through the phosphorylation of dopamine and cyclic-AMP-regulated phosphoprotein-32 (DARPP-32) $[13,14]$. Cdk 5 has been shown to regulate the neuregulininduced expression of acetylcholine receptors at the neu-

\section{KARGER}

Fax +41613061234 E-Mail karger@karger.ch www.karger.com (c) 2006 S. Karger AG, Basel

$1424-862 X / 06 / 0154-0157 \$ 23.50 / 0$

Accessible online at:

www.karger.com/nsg
Dr. Harish C. Pant

Cytoskeletal Protein Regulation Section, National Institute of Neurological Disorders and Stroke, National Institutes of Health, Building 49, Room 2A-35 Bethesda, MD 20892 (USA)

Tel. +1 301402 2124, Fax +1 301496 1339, E-Mail panth@ninds.nih.gov 
romuscular junction, the behavioral and adaptive changes associated with cocaine use as well as learning, memory and synaptic plasticity [15-17]. It is significant that $\mathrm{Cdk} 5$ plays a regulatory role since it is involved in 'crosstalk' with other kinase and signal transduction cascades. Cdk5 participates in 'cross-talk' with the extracellular signal-regulated kinase (Erk) pathway [18], calcium/ calmodulin-dependent protein kinase (CaMK) [19], cJun N-terminal kinase (JNK3) [20] pathways and, more recently, RasGRF2-mediated Rac signaling [21].

Our previous findings showed that p35/Cdk5 phosphorylation of the Ras guanine nucleotide exchange factor 2 (RasGRF2) resulted in regulation of MAP1b distribution in neurons through modulation of Rac-mediated Erk1/2 activity [21]. RasGRF1 and RasGRF2 are guanine nucleotide exchange factors (GEFs) that display a high degree of homology to each other, where RasGRF2 is ubiquitously expressed while RasGRF1 is mainly expressed in the nervous system. Thus, we wanted to investigate whether RasGRF1 was also a substrate of Cdk5 and what the biological function of this phosphorylation was in neurons. We found that RasGRF1 was phosphorylated by Cdk5. This phosphorylation on serine 731 regulated RasGRF1 steady state levels and tight regulation of RasGRF1 levels was critical for proper nuclear organization in both mitotic and post-mitotic cells. When RasGRF1 levels are elevated through the loss of Cdk 5 activity or by RasGRF1 overexpression, neuronal nuclei appeared condensed and fragmented. When RasGRF1 levels are reduced by increased Cdk 5 activity, the nuclei are also condensed and disorganized thus suggesting that nuclear organization was controlled by RasGRF1 levels which, in turn, was controlled by Cdk5 phosphorylation.

\section{Materials and Methods}

\section{Antibodies and Reagents}

Polyclonal anti-RasGRF1 antibody (Santa Cruz Biotechnology, Santa Cruz, Calif., USA) were used at 1:1,000 for immunocytochemistry in transfected cells, 1:500 for Western blotting, 1:100 for endogenous protein detection by immunocytochemistry and at 1:50 for immunoprecipitations. Anti-p35 (C19) and Cdk5 (J3 and C8) antibodies (Santa Cruz Biotechnology) were used at 1:1,000 dilutions to detect transfected proteins and at 1:200 to detect endogenous proteins. Monoclonal anti-Xpress (Invitrogen, Carlsbad, Calif., USA) and T7 (Novagen, San Diego, Calif., USA) epitope tag antibodies were used at 1:500 and 1:1,000 dilutions in Western blotting studies, respectively. Phospho-serine/threonine-proline (MPM-2) antibody was obtained from Upstate (Chicago, Ill., USA). Horseradish peroxidase-conjugated secondary antibodies (Amersham-Pharmacia, Piscataway, N.J., USA) were used at 1:2,000 dilutions. Anti-mouse/rabbit Oregon green, Texas red and Alexa Fluor 633 fluorescent-conjugated antibodies (Invitrogen, Molecular Probes, Eugene, Oreg., USA) were used at 1:400 dilutions. EGTA was purchased from Sigma (St. Louis, Mo., USA). Calpain I ( $\mu$-calpain) and II (m-calpain) inhibitors (N-acetylLeu-Leu-norleucinal and N-acetyl-Leu-Leu-methioninal, respectively) were obtained from Roche Biochemicals (Indianapolis, Ind., USA) and were described previously $[22,23]$.

\section{Plasmids}

p35, wild-type and dominant-negative $\mathrm{Cdk} 5 \mathrm{cDNAs}$ in pcDNA3.1C vector were cloned into pcDNA3.1C and described previously [21]. Rat RasGRF1 was cloned into pcDNA3 vector (Invitrogen). p35, Cdk5 and DNCdk5 were subcloned into the lentiviral vector packaging system and cortical neurons were transduced as previously described [24]. Wild-type Ras was subcloned into the pCEFL vector.

\section{Mammalian Cell Culture and Transfection}

$\mathrm{CHO}$ and COS cells were grown in F12/HAM and AdvancedDMEM, respectively (Invitrogen), containing 10\% (v/v) fetal bovine serum supplemented with $2 \mathrm{mM}$ glutamine, $100 \mathrm{U} / \mathrm{ml}$ penicillin and $100 \mu \mathrm{g} / \mathrm{ml}$ streptomycin. CHO and COS-7 cells were transfected using lipofectamine (Invitrogen) essentially according to the manufacturer's instructions. For immunofluorescence studies, COS cells were cultured in 6-well plates (BD Biosciences, Palo Alto, Calif., USA) on glass coverslips. Transfected CHO cells were treated with $2 \mathrm{mM}$ EGTA, $100 \mu \mathrm{M}$ calpain inhibitor I and $50 \mu \mathrm{M}$ calpain inhibitor II for $16 \mathrm{~h}$. Total cell lysates were prepared for Western blotting as described previously [25]. Rat and mouse embryonic cortical neurons were prepared using the papain disassociation technique described previously [24] and cultured for 3-7 days in culture (DIC) prior to analyses. Transfections of embryonic mouse and rat cortical neurons were performed after 3 DIC using lipofectamine 2,000 (Invitrogen).

\section{Co-Immunoprecipitation Studies}

$\mathrm{CHO}$ cells were transfected using lipofectamine (Invitrogen) according to the manufacturer's instructions with empty pcDNA3.1, RasGRF1, RasGRF1+p35 and RasGRF1+p35+Cdk5. Cells were harvested $24 \mathrm{~h}$ after transfection and lysed in ice-cold immunoprecipitation (IP) lysis buffer containing $50 \mathrm{mM}$ Tris- $\mathrm{Cl}$ $\mathrm{pH}$ 7.5, $150 \mathrm{~mm} \mathrm{NaCl}, 1 \%$ Triton-X-100, $1 \mathrm{~mm}$ EDTA, $5 \mu \mathrm{g} / \mathrm{ml}$ leupeptin, $2 \mu \mathrm{g} / \mathrm{ml}$ aprotinin, $5 \mu \mathrm{g} / \mathrm{ml}$ pepstatin, $1 \mathrm{mM}$ PMSF, $10 \mu \mathrm{M}$ sodium fluoride and $1 \mu \mathrm{M}$ sodium orthovanadate. Cells were incubated on ice for $30 \mathrm{~min}$, vortexed briefly and then cleared by centrifugation at $14,000 \mathrm{rpm}$ for $30 \mathrm{~min}$. Lysates were precleared for $1 \mathrm{~h}$ at $4{ }^{\circ} \mathrm{C}$ and RasGRF1 was immunoprecipitated using anti-RasGRF1 antibody for $2 \mathrm{~h}$ at $4^{\circ} \mathrm{C}$ with constant rocking. Immunoglobulin was pulled down using protein A Sepharose beads (Sigma) overnight at $4^{\circ} \mathrm{C}$. Immunoprecipitates were washed three times with IP lysis buffer. Samples were prepared for SDSPAGE analysis by addition of SDS sample buffer and heated at $95^{\circ} \mathrm{C}$ for $5 \mathrm{~min}$.

\section{Immunocytochemical Analyses}

Cortical neurons and transfected COS-7 cells grown on coverslips were fixed in $4 \%(\mathrm{w} / \mathrm{v})$ paraformaldehyde in PBS for $20 \mathrm{~min}$, permeabilized in $0.1 \%$ (v/v) Triton X-100/PBS for $20 \mathrm{~min}$, blocked with $5 \%(\mathrm{v} / \mathrm{v})$ fetal bovine serum $/ 0.1 \%(\mathrm{v} / \mathrm{v})$ Tween-20 in PBS for $1 \mathrm{~h}$, and then probed with primary antibodies diluted in 
blocking solution. To examine endogenous RasGRF1 staining, 5DIC cortical neurons were used. Primary antibodies were then visualized using either goat anti-mouse or goat anti-rabbit Igs coupled to Oregon green or Texas red (Molecular Probes), diluted in blocking solution for $1 \mathrm{~h}$ at room temperature and nuclei were counter-stained using DAPI (Sigma). Coverslips were washed in PBS between incubations and mounted in Gel/Mount ${ }^{\mathrm{TM}}$ (Biomeda, Hayward, Calif., USA). Fluorescence images were acquired using a $63 \mathrm{X}$ oil-immersion objective on a Zeiss LSM510 confocal microscope and managed with Adobe Photoshop.

\section{Immunohistochemical Analyses}

Immunohistochemistry of mouse brain sections was performed as described previously [26]. Briefly, 3-month-old female DLB7 mice were deeply anaesthetised with pentobarbitone and transcardially perfused with PBS followed by a freshly made and filtered solution of $4 \%$ paraformaldehyde in PBS ( $\mathrm{pH} 7.4$ ). Brains were subsequently removed and post-fixed for $6 \mathrm{~h}$ at $4^{\circ} \mathrm{C}$ in the same fixative prior to cryoprotection at $4^{\circ} \mathrm{C}$ in a solution of $30 \%$ sucrose in Tris-buffered saline (TBS: $40 \mathrm{~mm}$ Tris, $0.7 \% \mathrm{NaCl}$ in $10 \mathrm{mM}$ phosphate buffer, $\mathrm{pH} 7.8$ ) containing $0.05 \%$ sodium azide prior to freezing on dry ice and storage at $-80^{\circ} \mathrm{C}$. $30-\mu \mathrm{m}$ frozen coronal sections were collected in TBS-azide buffer at $4^{\circ} \mathrm{C}$ prior to immunohistochemical staining for RasGRF1 and p35. The optimal concentrations of primary (rabbit anti-p35 and RasGRF1, 1:200 and 1:100, respectively) were determined by performing a checkerboard titration. Sections were rinsed in TBS and blocked for 20 min with $15 \%$ normal goat serum (NGS) prior to incubation in a solution of anti-RasGRF1 and p35 antisera in TBS-T (TBS containing $0.3 \%$ Triton X-100) $/ 10 \% \mathrm{NGS}$ for $16 \mathrm{~h}$ at $4^{\circ} \mathrm{C}$. Sections were subsequently rinsed with TBS, incubated for $2 \mathrm{~h}$ in secondary antiserum (goat anti-rabbit IgG-Tx Red, Molecular Probes), diluted 1:400 in TBS-T/10\% NGS. Images were captured on a Zeiss LSM 510 confocal microscope. Experiments were repeated three times and identical results were obtained from all three experiments and representative sections were used for illustration.

\section{In vitro Site-Directed Mutagenesis}

RasGRF1 serine-alanine 731 phosphorylation mutant (RasGRF1 $1^{\text {Ser-Ala731 }}$ ) was produced using the Quikchange XL sitedirected mutagenesis kit (Stratagene, La Jolla, Calif., USA) according to the manufacturer's instructions using wild-type RasGRF1/pCEFL as the template. Putative mutants were sequenced to confirm the mutation of serine 731 to alanine 731 using primers 50 bases upstream and downstream of the site.

In vitro and in vivo Phosphorylation of RasGRF1 by p35/Cdk5 $\mathrm{CHO}$ cells were transfected with RasGRF1 and RasGRF1 ${ }^{\text {Ser-Ala731 }}$. RasGRF1 was immunoprecipitated using the polyclonal anti-RasGRF1 antibody. Immunoprecipitates were incubated with GST, GST-Cdk5+GST-p35 [27] and $\gamma^{32}$ P-ATP with and without roscovitine, separated by SDS-PAGE and transferred onto nitrocellulose membranes. Membranes were exposed to the STORM phosphoimager followed by immunodetection of RasGRF1 using Western blotting. To confirm Cdk5 activity, histone $\mathrm{H} 1$ was used as a substrate in identical reactions described above. Samples were resolved by SDS-PAGE and gels were Coomassie stained and destained then dried and subjected to autoradiography. RasGRF1 was immunoprecipitated from wild-type and p35 knockout mouse brain lysates, separated by SDS-PAGE and sub- jected to Western blotting using anti-RasGRF1 and anti-phospho-serine antibodies to detect total and phosphorylated RasGRF1, respectively.

Ras and Rac Activity Assays

Ras assays were performed using the EZDetect Ras Activation Kit according to the manufacturer's instructions (Pierce, Rockford, Ill., USA). CHO cells were transfected with empty vector+Ras, RasGRF1+Ras, RasGRF1+Ras+p35+Cdk5, RasGRF1+Ras+p35+dominant negative Cdk5 and Ras$\mathrm{GRF} 1^{\text {Ser-Ala731 }}+\mathrm{p} 35+\mathrm{Cdk} 5$. After $24 \mathrm{~h}$, cells were harvested using ice-cold IP lysis buffer containing $10 \mathrm{mM} \mathrm{MgCl}_{2}$. Active Ras levels were assayed by incubation with GST-c-Raf on glutathione Sepharose beads for $1 \mathrm{~h}$. Rac activity assays were performed as described previously with the use of the EZDetect Rac activity kit using GST-PAK1-BD as bait (Pierce). Bound active Ras and Rac were washed three times in lysis buffer and eluted from the GST beads using $2 \times$ SDS sample buffer containing $5 \% \beta$-mercaptoethanol (Sigma) and boiling for $5 \mathrm{~min}$. Samples were separated using 4-20\% SDS-PAGE and subjected to Western blotting using the monoclonal anti-Ras and Rac antibodies provided in the kit. Ras and Rac activity were determined by detecting Ras and Rac eluted from GST-c-Raf/PAK1-BD beads and normalizing this signal to that of total Ras and Rac found in lysates.

\section{Results}

\section{p35 Interacts with RasGRF1}

In a previous study from our laboratory, we reported that RasGRF2 interacted with and was phosphorylated by $\mathrm{p} 35 / \mathrm{Cdk} 5$. We wanted to know whether the homologous protein RasGRF1 also interacted with $\mathrm{p} 35 / \mathrm{Cdk} 5$ and used co-immunoprecipitation assays to investigate this. In $\mathrm{CHO}$ cells that were co-transfected with RasGRF1 with and without p35/Cdk5, it was found that p35 co-immunoprecipitated with RasGRF1 in these studies (fig. 1A). This is consistent with our previous findings, and of others, that showed p35 directs substrates to Cdk5 $[5,25,28]$. Interestingly, we also found that upon cotransfection of RasGRF1+p35+Cdk5, RasGRF1 steady state levels were consistently reduced, a result expanded later in the study. To determine whether p35 and RasGRF1 did indeed exist in similar cellular compartments and to further confirm the co-immunoprecipitation data, we transfected COS-7 cells with p35+RasGRF1 and p35+Cdk5+RasGRF1. Both p35 and RasGRF1 existed in identical cytoplasmic compartments in transfected COS7 cells and co-localized with each other (fig. 1Bd, h). To confirm the interaction in brain lysates, p35 was immunoprecipitated from rat brain lysates using the monoclonal anti-p35 antibody and immunodetected using C19 polyclonal antibody (fig. 1C, top panel). RasGRF1 was present in this immunoprecipitate (fig. 1C, bottom pan- 


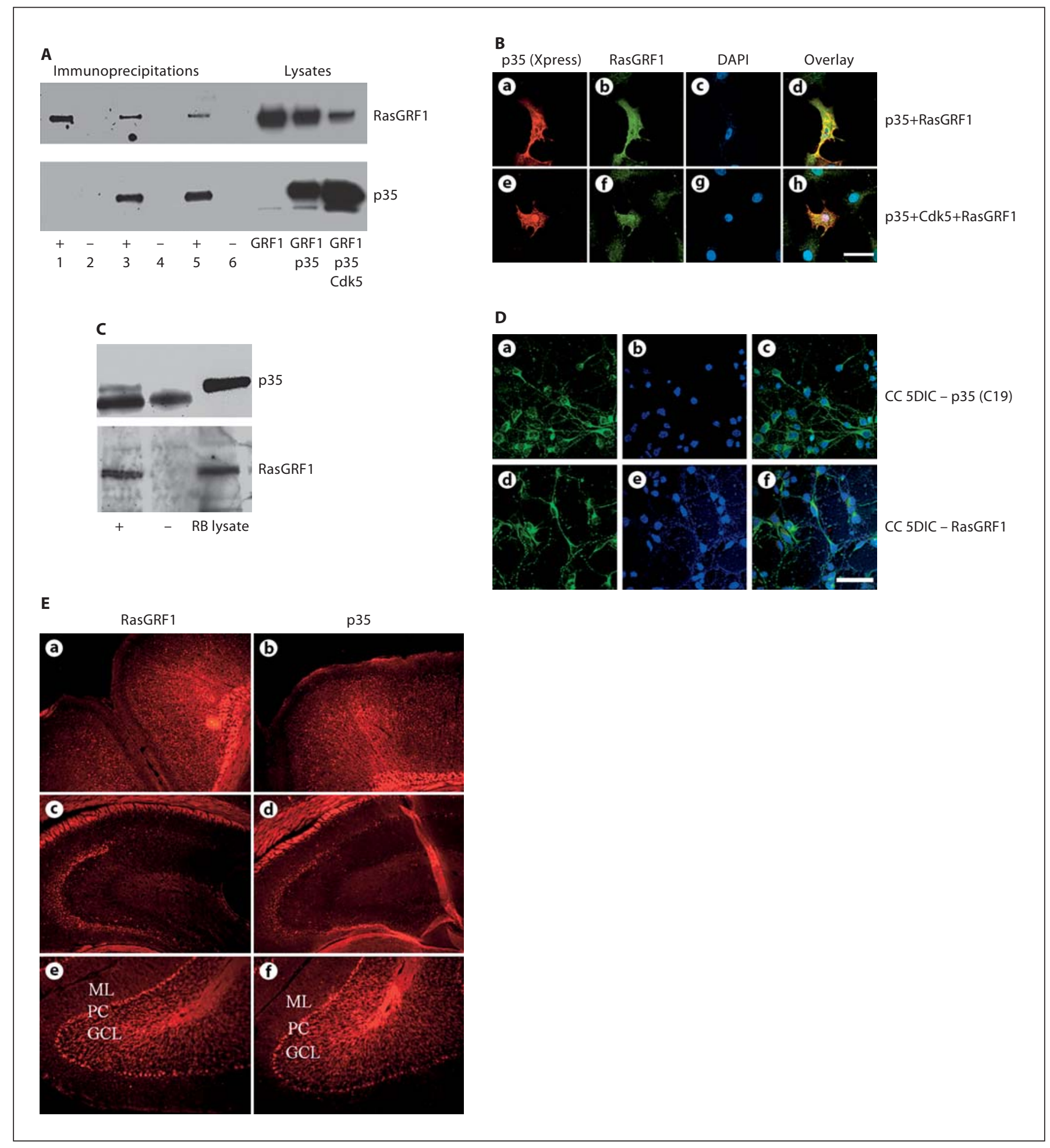

Fig. 1. p35 associates with RasGRF1. A CHO cells were transfected with RasGRF1 only (lanes 1 and 2), RasGRF1+p35 (lanes 3 and 4) and RasGRF1+p35+Cdk5 (lanes 5 and 6). RasGRF1 was immunoprecipitated and immunodetected using the polyclonal anti-RasGRF1 antibody and p35 immunodetected using monoclonal anti-Xpress antibody. + and - refer to the presence or ab- sence of immunoprecipitating antibody, respectively. In both RasGRF1+p35 and RasGRF1+p35+Cdk5 transfected cells, p35 co-immunoprecipitated with RasGRF1. B COS-7 cells were transfected with RasGRF1+p35 and RasGRF1+p35+Cdk5 and subjected to immunofluorescence. p35 was visualized using the monoclonal anti-Xpress antibody and RasGRF1 was visualized using 
el). To determine the endogenous distribution of RasGRF1 and p35 in neurons, we immunostained 5 days in culture (5-DIC) cortical neurons with polyclonal p35 and RasGRF1 antibodies. We found that p35 and RasGRF1 occupy similar perikaryal distributions in 5-DIC cortical neurons (fig. 1Da-f). Immunohistochemical staining of parallel mouse brain sections showed that RasGRF1 and p35 occupy similar areas of the brain. Both proteins are found in the retrosplenial granular areas of the cortex (fig. 1Ea-b), the CA 2 and 3 regions of the hippocampus (fig. $1 \mathrm{Ec}-\mathrm{d}$ ) and regions of the cerebellum such as the Purkinje cell layer (PC) and the granule cell layer (GCL). Both proteins are, however, not found in the molecular layer (ML) (fig. 1Ee-f).

\section{Cdk5 Phosphorylates RasGRF1 on Serine 731}

Our previous study showed that $\mathrm{p} 35 / \mathrm{Cdk} 5$ phosphorylated RasGRF2 on serine 737 and mediated Rac signaling and Erk1/2 activity [21]. The corresponding residue in RasGRF1 is serine 731, where the amino acid sequence is TSS*PVRRR, an ideal consensus Cdk5 phosphorylation site. To determine whether RasGRF1 is a substrate of Cdk5, RasGRF1 wild-type and phospho-deficient mutant RasGRF1 ${ }^{\text {Ser-Ala731 }}$ were immunoprecipitated from transfected $\mathrm{CHO}$ cells and used in in vitro kinase assays using recombinantly expressed and purified GST and GST-p35/Cdk5 in the absence and presence of the potent Cdk5 inhibitor roscovitine. RasGRF1 was phosphorylated when incubated with GST-p35/Cdk5 but not when incubated with GST only (fig. 2B, lanes 1 and 2). This phosphorylation was inhibited in the presence of $10 \mu \mathrm{M}$ rosco-

the polyclonal anti-RasGRF1 antibody. Overlay panels show RasGRF1 and p35 co-localization in yellow. C p35 was immunoprecipitated from rat brain lysate using monoclonal anti-p35 antibody and p35 and RasGRF1 immunodetected using anti-C19 (top panel) and RasGRF1 (bottom panel) polyclonal antibodies, respectively. + and - refer to the presence or absence of immunoprecipitating antibody, respectively, and rat brain lysate resolved as a marker of the proteins (RB lysate). p35 (indicated by the asterisk) co-immunoprecipitated with RasGRF1. D To determine whether endogenous $\mathrm{p} 35$ and RasGRF1 exist in similar compartments in neurons, 5 days in culture (5DIC) cortical neurons were immunostained for the presence of p35 (a) and RasGRF1 (d), nuclei staining $(\mathbf{b}, \mathbf{e})$ and overlays are shown in $\mathbf{c}$ and $\mathbf{f}$. Both proteins exhibited perikaryal localization in neurons. Scale bar $=50 \mu \mathrm{m}$. E To determine whether RasGRF1 and p35 were similarly distributed in adult mouse brain, coronal sections were immunostained using polyclonal RasGRF1 (a, c, e) and p35 (b, d, f) antibodies. RasGRF1 and p35 occupied similar areas in the cortex, hippocampus and cerebellum.

p35/Cdk5 Phosphorylates RasGRF1 vitine (fig. 2B, lane 3). GST-p35/Cdk5 was unable to phosphorylate RasGRF1 ${ }^{\text {Ser-Ala731 }}$ confirming that this was indeed the site of $\mathrm{p} 35 / \mathrm{Cdk} 5$ phosphorylation (fig. 2B, lane 4). To confirm the activity of the GST-Cdk5/p35 complex, histone was used as a substrate in kinase assays with and without roscovitine. Histone phosphorylation was absent when incubated with GST only (fig. 2C, lane 1) as well as when incubated in the presence of roscovitine (fig. 2C, lane 3) and highly phosphorylated with GSTCdk5/p35 (fig. 2C, lane 2). To confirm the in vitro data, we immunoprecipitated RasGRF1 from age-matched wild-type and p35 knockout (p35-/-) mouse brain lysates. The level of serine/threonine-proline phosphorylation of RasGRF1 was decreased in p35-/- lysates compared to wild-type lysates (fig. 2D, top panel). Additionally, the expression of RasGRF1 was increased in p35-/- brain (see below).

\section{Cdk5 Controls RasGRF1 Steady State Levels}

It was found that steady state levels of RasGRF1 in p35-/- lysates were elevated compared to wild-type lysates (fig. 3A, top panel) while Cdk5levels were unchanged (data not shown). To confirm that this effect was attributed to Cdk5 activity in neurons, cortical neurons were transduced with lentiviral combinations of empty vector (EV), p35+Cdk5 and p35+dominant-negative Cdk5. The overexpression of $\mathrm{p} 35+\mathrm{Cdk} 5$ caused a reduction in RasGRF1 levels compared to EV infected neurons (fig. 3B). The infection of p35+dominant-negative Cdk5 rescued RasGRF1 levels (fig. 3B, lane 3). To identify the precise site that is phosphorylated by Cdk5 to control RasGRF1 levels, CHO cells were co-transfected with RasGRF1, RasGRF1+p35+Cdk5, RasGRF1+p35+ dominant negative Cdk5 and RasGRF1 $1^{\text {Ser-Ala731 }}+$ p35+Cdk5. The co-transfection of active Cdk5 with wild-type RasGRF1 (fig. 3C, lane 2) led to a reduction in RasGRF1 levels compared to RasGRF1-only transfected cells (fig. 3C, lane 1). Additionally, the co-transfection of RasGRF1 with p35+dominant negative Cdk5 (fig. 3C, lane 3) and the co-transfection of RasGRF1 ${ }^{\text {Ser-Ala731 }}$ with p35+Cdk5 (fig. 3C, lane 4) rescued the levels of RasGRF1. These data suggested that phosphorylation of RasGRF1 leads to its degradation.

\section{RasGRF1 Is Proteolyzed by m-Calpain upon Cdk5 Phosphorylation}

To elucidate the mechanism of RasGRF1 degradation, we treated RasGRF1+p35+Cdk5-transfected cells with the proteosomal inhibitor MG132 and the lysosomal inhibitor ammonium chloride $\left(\mathrm{NH}_{4} \mathrm{Cl}\right)$. We found that neither inhibitor could prevent the degradation of RasGRF1. 


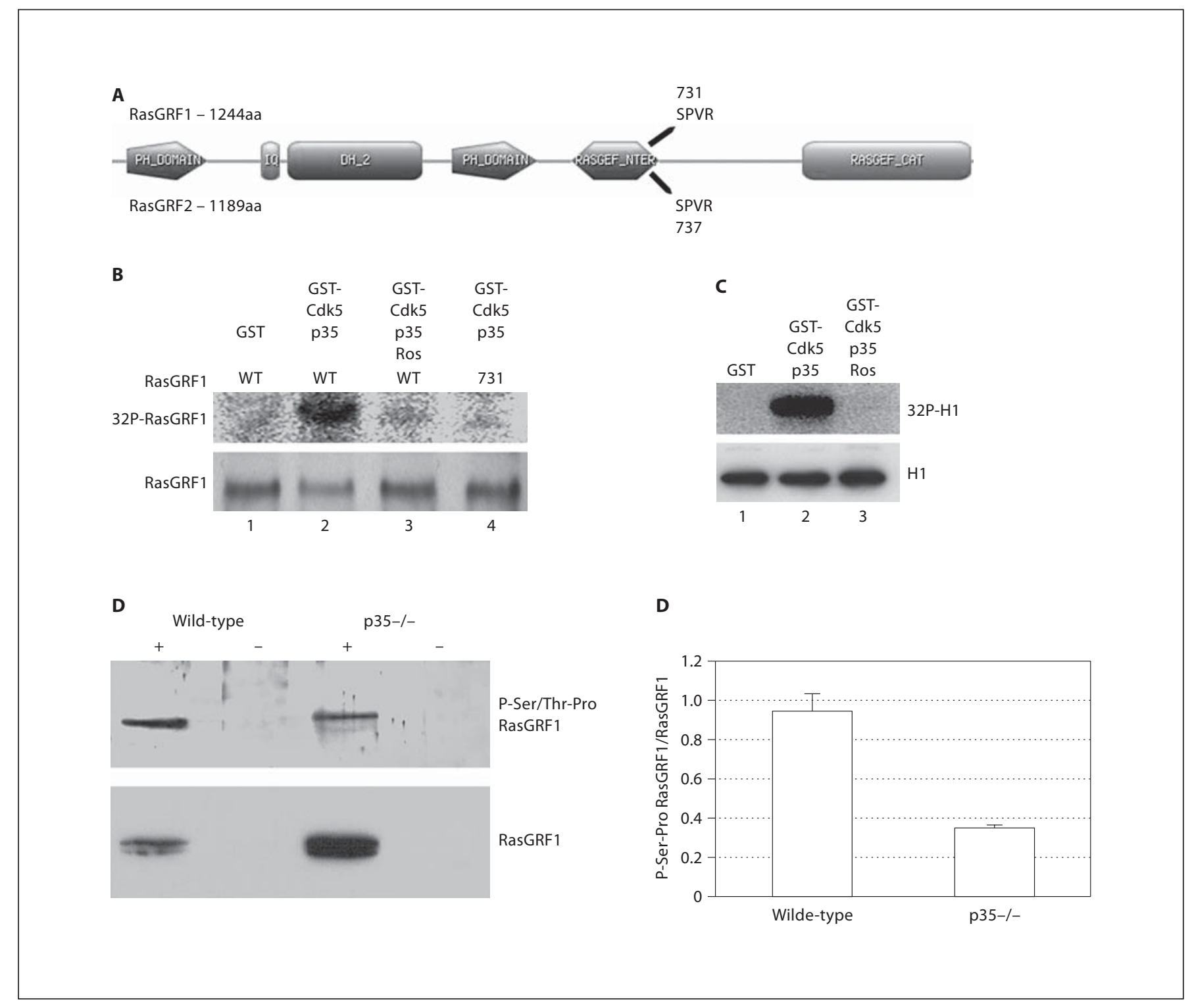

Fig. 2. Cdk5 phosphorylates RasGRF1. A Schematic representation of the Ras guanine nucleotide exchange factors (RasGEFs) RasGRF1 and RasGRF2 showing the common domains. $\mathrm{PH}=$ Pleckstrin homology; IQ = calcium-calmodulin binding domain; $\mathrm{DH}=$ Dbl homology domain; NTER = amino-terminal domain; $\mathrm{CAT}=$ catalytic domain. Figure indicates the published Cdk5 phosphorylation site on RasGRF2 (serine 737) and the corresponding site (serine 731) in RasGRF1. B In vitro kinase assays were performed in the presence and absence of the specific Cdk5 inhibitor roscovitine (Ros) to confirm the site of phosphorylation using recombinant expressed GST, GST-p35+GST-Cdk5. CHO cells were transfected with RasGRF1 (WT) or with RasGRF1 serine $^{731}$-alanine $\mathrm{e}^{731}$ (731) mutant, immunoprecipitated and used as the substrate. Samples were separated by SDS-PAGE and transferred onto nitrocellulose. The autorad (top panel) was obtained prior to RasGRF1 immunodetection using anti-RasGRF1 antibody (bottom panel) where Cdk5/p35 phosphorylated RasGRF1 ${ }^{32} \mathrm{P}-\mathrm{R}$ asGRF1) and this was abolished with the serine-alanine 731 mutant (731). C As the positive control for Cdk5/p35 activity, histone $\mathrm{H} 1$ was used as a substrate in similar assays. Autorad (top panel) shows the phosphorylated histone $\mathrm{H} 1\left({ }^{32} \mathrm{P}-\mathrm{H} 1\right)$ while the corresponding Coomassie-stained gel (bottom panel) shows equal loading of histone $\mathrm{H1}$ (H1). Results shown are a representative autroradiograph of three separate experiments with identical results. D Lysates were prepared from adult wild-type and p35 knockout (p35-/-) mice brain. RasGRF1 was immunoprecipitated and the presence of phospho-RasGRF1 was immunodetected using the phospho-serine/threonine-proline antibody (top panel). The presence of total RasGRF1 was confirmed using the RasGRF1 antibody (bottom panel). The phosphorylation was quantified in the bar graph on the right where phospho-serine/threonine-proline-RasGRF1 signal was normalized to total RasGRF1. Results are expressed as \pm SEM of 4 independent experiments. There was an approximate 2.5 -fold reduction in phospho-RasGRF1 in the Cdk5 knockout compared to wild-type immunoprecipitates. 
Fig. 3. Cdk5 phosphorylation controls RasGRF1 steady state levels. A Lysates from age-matched wild-type (WT) and p35 knockout mice (p35-/-) were separated by SDS-PAGE and transferred onto nitrocellulose and the presence of RasGRF1 was immunodetected using RasGRF1 antibody. Equal loading was confirmed by immunodetection of tubulin. p35 knockout mice lysates showed elevated steady state RasGRF1 levels and quantification is shown in the corresponding bar graph. B Lysates from lentiviral-infected rat cortical neurons were resolved by SDS-PAGE, transferred onto nitrocellulose and immunodetected using Cdk5, p35 and RasGRF1 antibodies. Neurons were infected with empty vector (EV), p35+wild-type Cdk5 (p35/WT) and p35+dominant-negative Cdk5 (p35/DN). Equal loading of lysates was confirmed by immunodetection of tubulin. Quantitation of RasGRF1 is shown in the corresponding bar graph where overexpression of p35 and wild-type Cdk5 reduced RasGRF1 levels by $50 \%$. Expression of p35 and dominant-negative Cdk5, rescued RasGRF1 levels. C CHO cells were transfected with (1) RasGRF1 (GRF1); (2) p35+Cdk5+RasGRF1; (3) p35+DNCdk5, and (4) p35+Cdk5+RasGRF1 serine ${ }^{731}$ alanine $^{731}$ mutant (GRF1 Ala 731) and lysates were separated by SDS-PAGE, transferred onto nitrocellulose. Equal loading of lysates was confirmed by actin immunodetection. Transfection of p35+Cdk5+RasGRF1 caused a 3-fold reduction in RasGRF1 levels that was rescued when dominant-negative Cdk5 and the RasGRF1 serine ${ }^{731}$-alanine $e^{731}$ mutant was used. Quantification of the data is shown in the corresponding bar graph on the right where results are expressed as \pm SEM of three separate experiments. D CHO cells were transfected with RasGRF1 (GRF1) and RasGRF1+p35+Cdk5 and the latter was treated with vehicle (UT) and 2 mM EGTA. EGTA-treated cells did not exhibit the p35/Cdk5-mediated degradation of RasGRF1. E CHO cells were transfected as described in (D) and cells were treated with vehicle (UT), $100 \mu \mathrm{M}$ calpain inhibitor I (CI) and $50 \mu \mathrm{M}$ calpain inhibitor II (CII). The degradation of RasGRF1 was inhibited when cells were treated with CII.
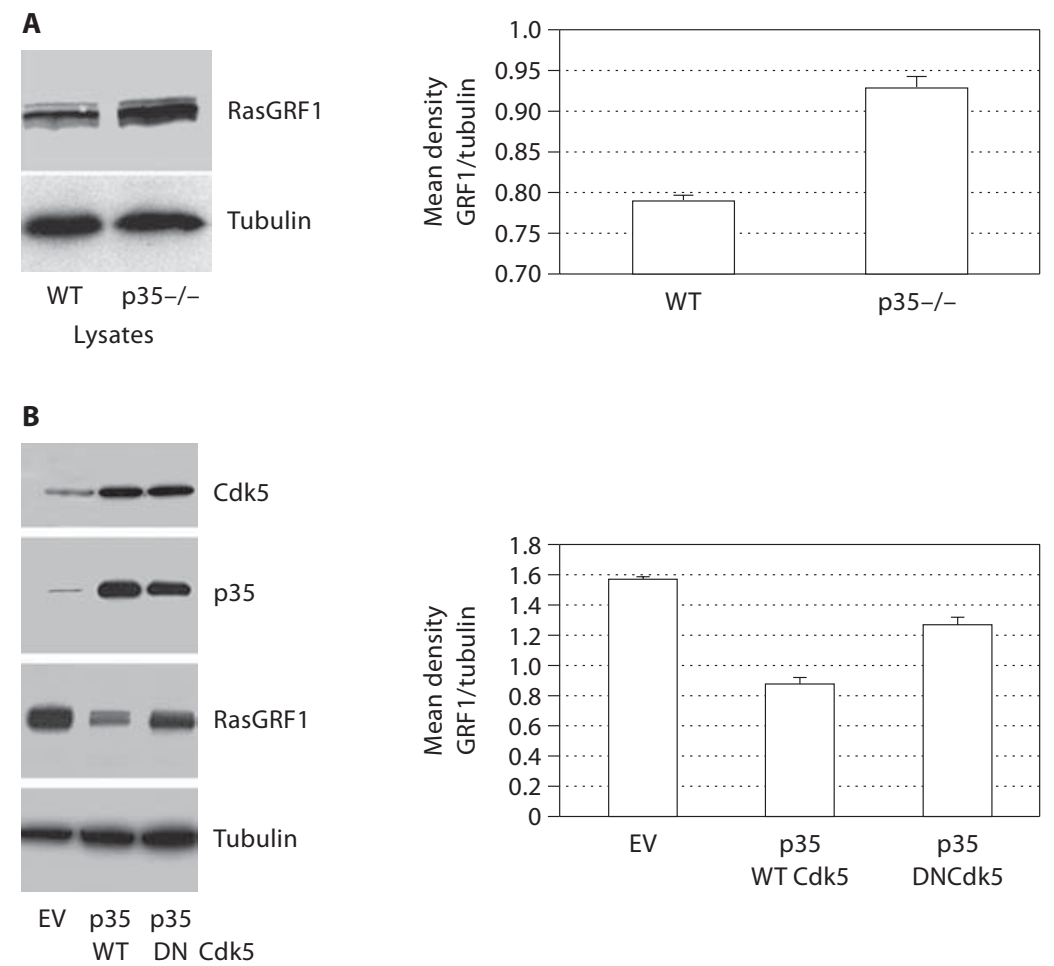

C
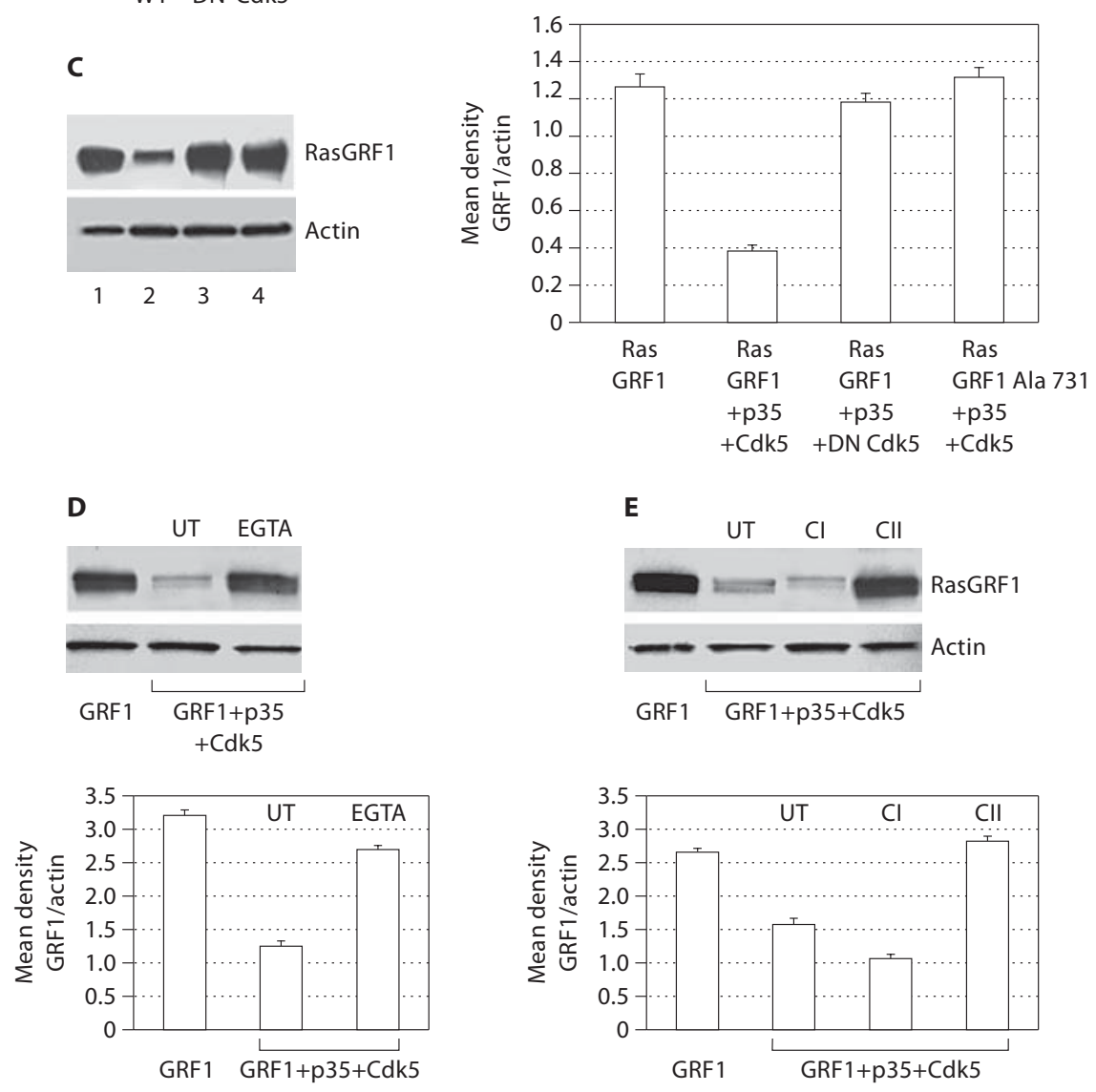
This suggested that degradation of RasGRF1 was not due to proteosomal or lysosomal pathways (data not shown). The other possible mechanism which may be responsible for phosphorylated RasGRF1 degradation was a calciumdependent proteolytic pathway. To evaluate this we treated RasGRF1+p35+Cdk5-transfected CHO cells with the calcium chelator EGTA and found a marked rescue of RasGRF1 levels (fig. 3D). We then tested calpain inhibitors I and II, which are sensitive for $\mu$-calpain and $\mathrm{m}$-calpain, respectively. Only the $\mathrm{m}$-calpain inhibitor (inhibitor II) was effective in rescuing RasGRF1 levels (fig. 3E).

\section{Cdk5 Regulates RasGRF1 Levels in Neurons}

To determine whether RasGRF1 levels are indeed regulated by Cdk5 in vivo, we performed immunocytochemical analyses of mouse embryonic cortical neurons. Cortical neurons from E16.5 wild-type and Cdk5 knockout mice were grown for 3 days in culture (DIC) before immunostaining for RasGRF1. This length of time was chosen since after this time, Cdk5 knockout neurons undergo massive cell death (data not shown, and [20]). Confocal images from Cdk5 knockout neurons (fig. 4Ad-f) contained greater RasGRF1 levels compared to the wildtype neurons (fig. $4 \mathrm{Aa}-\mathrm{c}$ ). These data corroborate data shown in figure 3B. To confirm this, we transfected wildtype Cdk5 into the knockout neurons and found that the Cdk5 knockout neurons containing wild-type Cdk5 (arrowed in fig. 4B) had reduced RasGRF1 immunostaining compared to surrounding nontransfected neurons. Additionally, nuclear organization of these neurons appeared normal compared to the condensed nuclei of neighboring, nontransfected neurons. To ensure that the levels of RasGRF1 are indeed controlled by Cdk5, we transfected dominant-negative $\mathrm{Cdk} 5$ into rat embryonic cortical neurons and found that the transfected neuron, without active Cdk5, exhibited increased RasGRF1 (fig. 4C) accompanied with altered nuclear morphology.

\section{Cdk5 Phosphorylation of RasGRF1 Causes Nuclear Disorganization}

To examine the effect of increasing or reducing RasGRF1 levels in cells, COS-7 cells were transfected with Ras only, Ras+RasGRF1+p35+Cdk5, Ras+ RasGRF1mut731+p35+Cdk5 and Ras+p35+Cdk5. Overexpression of RasGRF1+Ras altered nuclear organization where nuclei were compacted (fig. $5 \mathrm{e}-\mathrm{h}$ ). The elevation of RasGRF1 levels through the expression of the RasGRF1 ${ }^{\text {Ser-Ala731 }}$ also caused similar nuclear changes (fig. $5 \mathrm{~m}-$ p). The nuclei of Ras-only- and p35+Cdk5+Rastransfected cells were not disorganized (fig. $5 \mathrm{a}-\mathrm{d}$ and
$5 q-t$, respectively), thus ruling out any effect attributed to the presence of $\mathrm{p} 35 / \mathrm{Cdk} 5$ or Ras.

\section{RasGRF1 Levels Are Essential for Neuronal Nuclear Organization}

Cdk5 knockout neurons, after 3 days in culture, exhibit condensed nuclei and start to die after this time (data not shown). RasGRF1 starts to be expressed at 3DIC in cortical neurons and so the question arose whether the loss of RasGRF1 regulation through the loss of Cdk 5 activity was the cause for the nuclear disorganization in these neurons. Experiments using transfection of dominant-negative C $\mathrm{dk} 5$ into neurons resulted in an increase in RasGRF1 coupled with nuclear condensation (arrowed in fig. 6Aa-d). Overexpression of $\mathrm{p} 35 / \mathrm{Cdk} 5$ in neurons also causes disintegration of neuronal nuclei with a concomitant decrease in RasGRF1 levels in the transfected neuron (arrowed in fig. $6 \mathrm{Be}-\mathrm{h})$. The data suggested that a loss of regulation of RasGRF1 levels, either through increases or decreases, is detrimental to neuronal nuclear organization. Since Cdk5 activity is involved in a number of neuronal functions, we needed to narrow down the role of RasGRF1 in controlling nuclear organization. For this, RasGRF1 ${ }^{\text {Ser-Ala731 }}$ was overexpressed so that it could not be degraded by Cdk5. In the transfected neuron (arrowed in fig. 6Ci-k), the build-up of RasGRF1 was clearly evident, as was the fragmentation of the nucleus of that neuron. Similar results were obtained when wild-type RasGRF1 was transfected into neurons suggesting that endogenous $\mathrm{p} 35 / \mathrm{Cdk} 5$ activity is insufficient to phosphorylate the elevated levels of RasGRF1 and the resultant over-expression causes similar nuclear changes (arrowed in fig. 6Dl-n).

\section{Phosphorylation of RasGRF1 by p35/Cdk5 Modulates Ras Signaling and Akt Activation}

Since RasGRF1 has been shown to activate Ras and Rac signaling pathways, the downstream effect of Cdk5 phosphorylation on RasGRF1 was investigated. Ras and Rac activity assays were performed using transfected $\mathrm{CHO}$ cells since these cells exhibited the highest transfection efficiencies of all cells tested. Ras only transfected cells showed moderately active Ras levels which increased when RasGRF1 was co-transfected with Ras (fig. 7A, lanes 1 and 2). The active Ras levels were reduced when RasGRF1 was co-transfected with p35+Cdk5+Ras (fig. 7A, lane 3). The presence of dominant-negative Cdk5+p35+RasGRF1 and the phospho-mutant Ras$\mathrm{GRF} 1^{\mathrm{Ser}-\mathrm{Ala} 731}+\mathrm{p} 35+\mathrm{Cdk} 5$ maintained the levels of active Ras comparable to the assays that contained only wildtype RasGRF1+Ras (fig. 7A, lanes 4 and 5). 

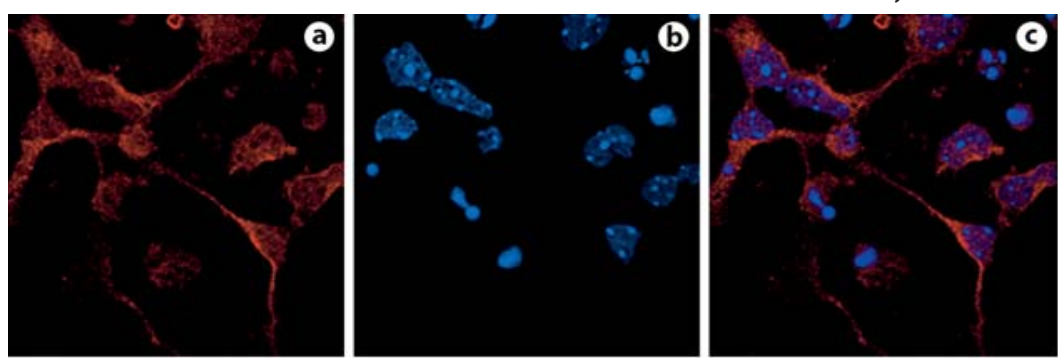

Wild type
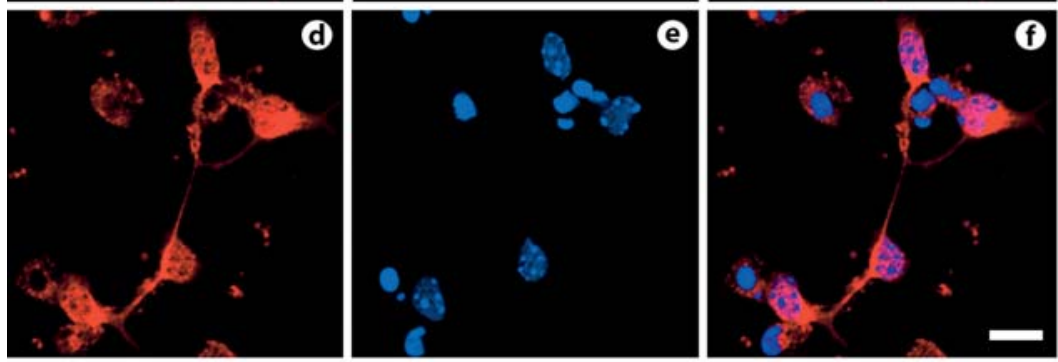

๑

Cdk5 knockout

B
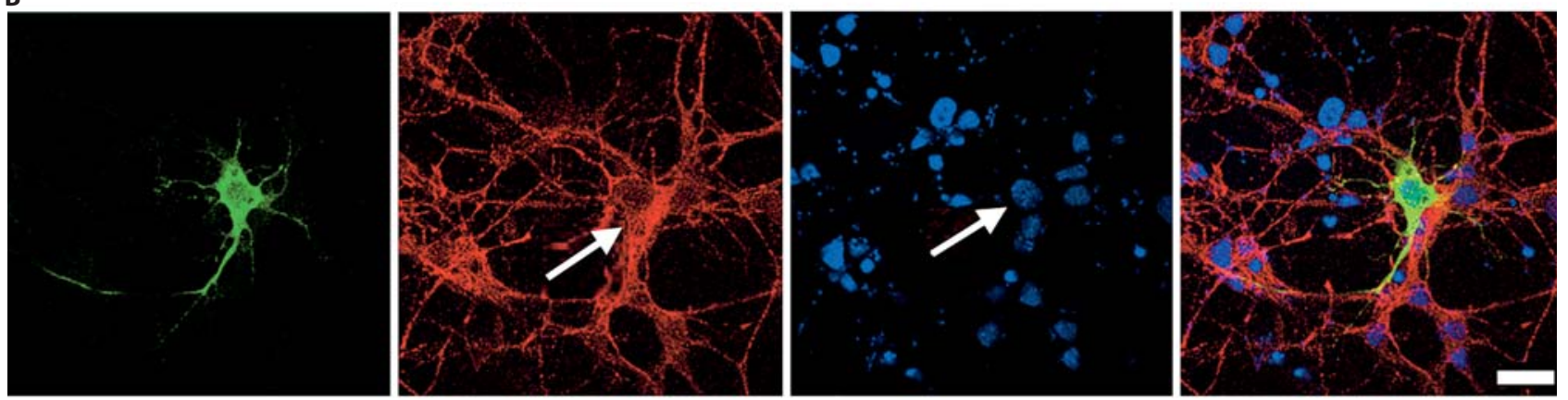

C
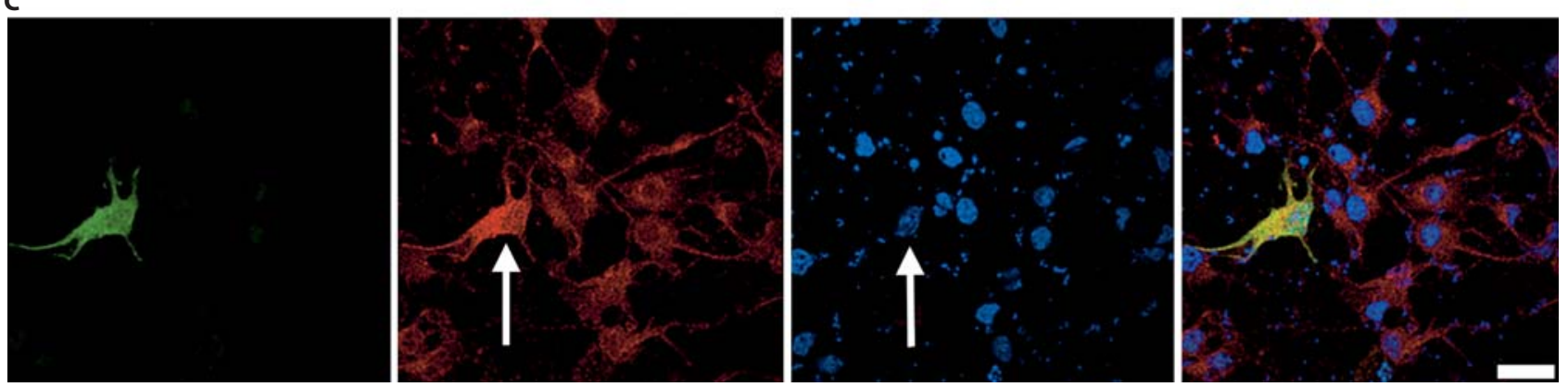

Fig. 4. Cdk5 regulates RasGRF1 levels in neurons. A E16.5 wildtype and Cdk5 knockout cortical neurons were plated and immunostained for RasGRF1 after 3 days in culture. Cdk5 knockout neurons showed increased RasGRF1 staining compared to agematched wild-type neurons. B 3 days in culture Cdk5 knockout mouse cortical neurons were transfected with wild-type Cdk5 and RasGRF1 levels were examined in the transfected neurons. The arrow indicates a transfected neuron in close proximity to a nontransfected neuron. The level of RasGRF1 is reduced in the transfected neuron compared to the surrounding nontransfected neurons. C 3DIC rat cortical neurons were transfected with dominant-negative Cdk5 (DNCdk5) and RasGRF1 levels were examined by immunostaining. The transfected neuron expressing DnCdk5 (arrowed) showed an increase in RasGRF1 compared to the surrounding nontransfected neurons. Scale bars $=20 \mu \mathrm{m}$. 


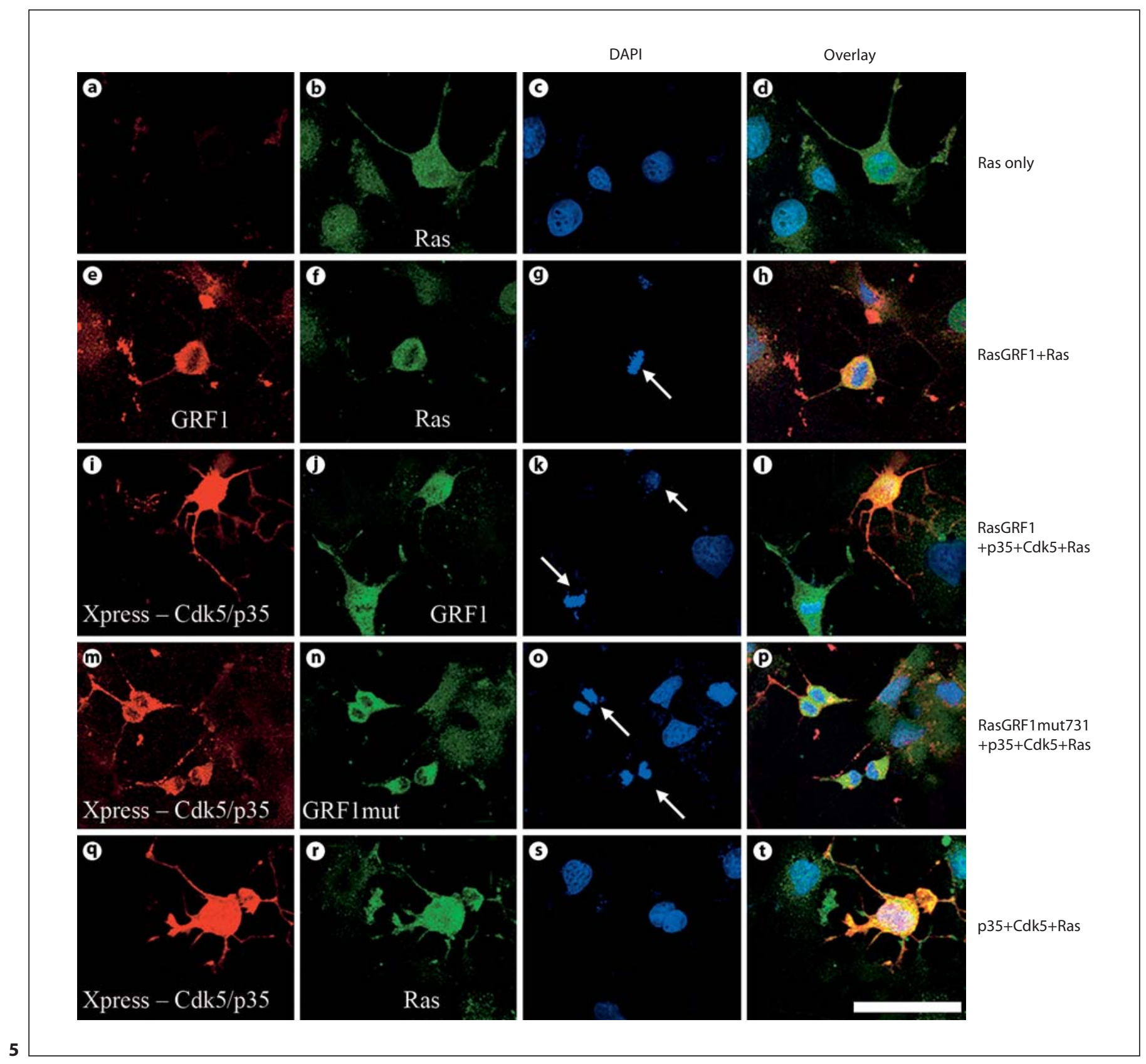

Fig. 5. Cdk5 phosphorylation of RasGRF1 causes nuclear disorganization in COS-7 cells. COS-7 cells were transfected with the following constructs: Ras only; where Ras was detected using monoclonal anti-AU5 epitope antibody and Oregon green (a-d). Ras+RasGRF1; where Ras was detected using Oregon green and RasGRF1 with Texas red (e-h). Ras+p35+Cdk5+RasGRF1 (i-l) and Ras+p35+Cdk5+RasGRF1mut731 (m-p) where p35/Cdk5 were detected using Xpress and Texas red and RasGRF1 with Oregon green. Ras+p35+Cdk5 (q-t); where Ras was detected using anti-AU5 epitope antibody and Texas red, while p35/Cdk5 was detected using anti-Xpress epitope tag and Texas red. Overexpression of RasGRF1+Ras altered nuclear organization and the elevation of RasGRF1 levels through the expression of the RasGRF1 $1^{\text {Ser-Ala731 }}$ also caused the nuclear changes. Scale bar $=$ $50 \mu \mathrm{m}$.

Fig. 6. Tight regulation of RasGRF1 levels is required for neuronal nuclear organization. A 3 days in culture rat cortical neurons were transfected with DNCdk5 and immunostained for Cdk5 and RasGRF1. Nuclei were stained with DAPI. In the transfected neuron, the RasGRF1 level was increased and its nucleus was condensed compared to the non-transfected neuron. B 3DIC rat cortical neurons were co-transfected with p35 and Cdk5. Transfected neurons were visualized with the T7 epitope tag antibody and nuclei were visualized with DAPI. The overexpression of 


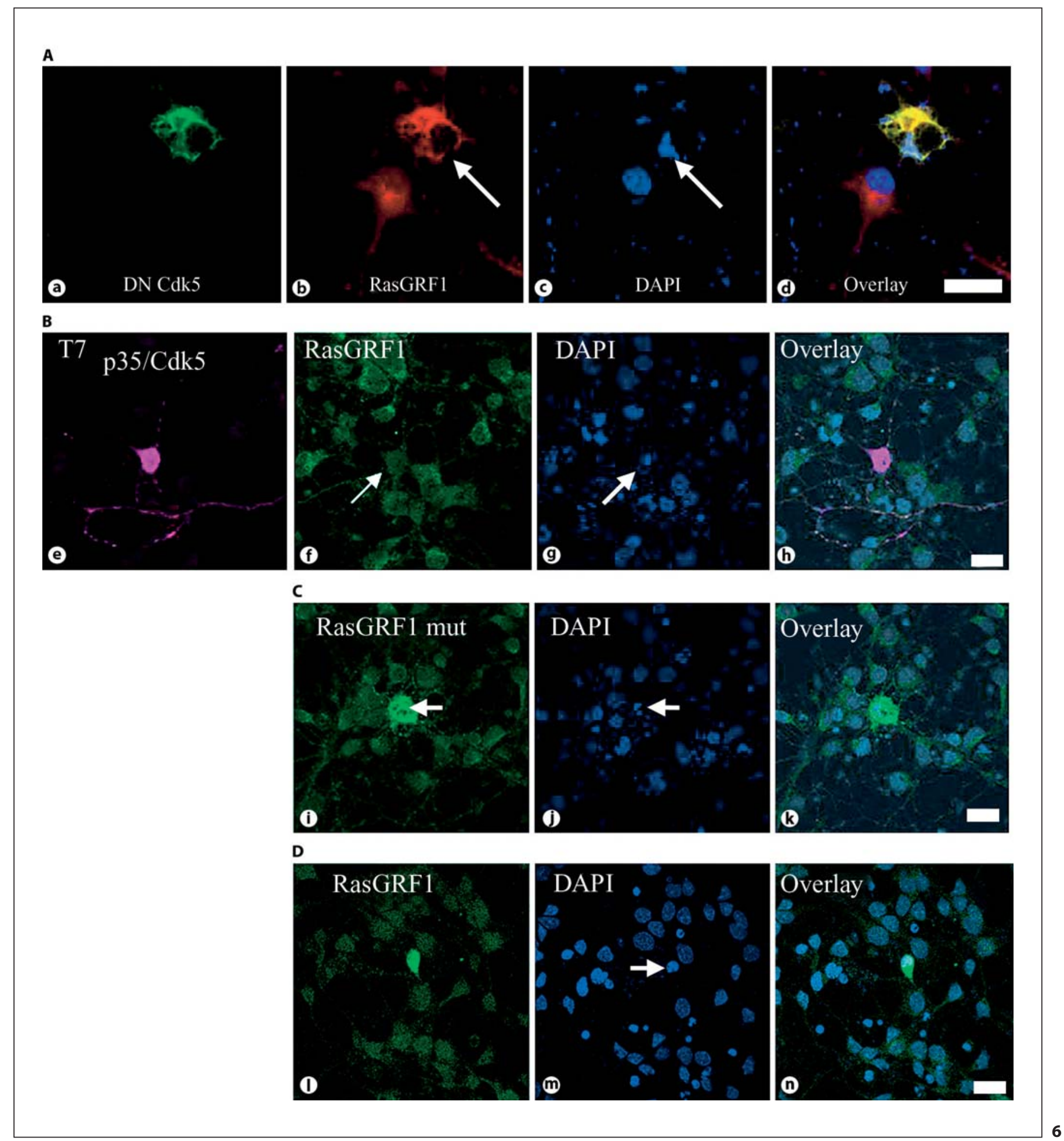

p35 and Cdk5 caused a similar disorganization of the nucleus accompanied with a reduction in RasGRF1 levels in the transfected neuron (arrowed). C 3DIC rat cortical neurons were transfected with RasGRF1 ${ }^{\text {Ser-Ala731 }}$ mutant and immunostained while nuclei were stained with DAPI. The increase in RasGRF1 levels overexpression corresponded with disintegration of the nucleus. D 3 days in culture rat cortical neurons were transfected with wild-type RasGRF1 immunostained while nuclei were stained with DAPI. The neuron overexpressing RasGRF1 showed an altered and shrunken nuclear morphology. 


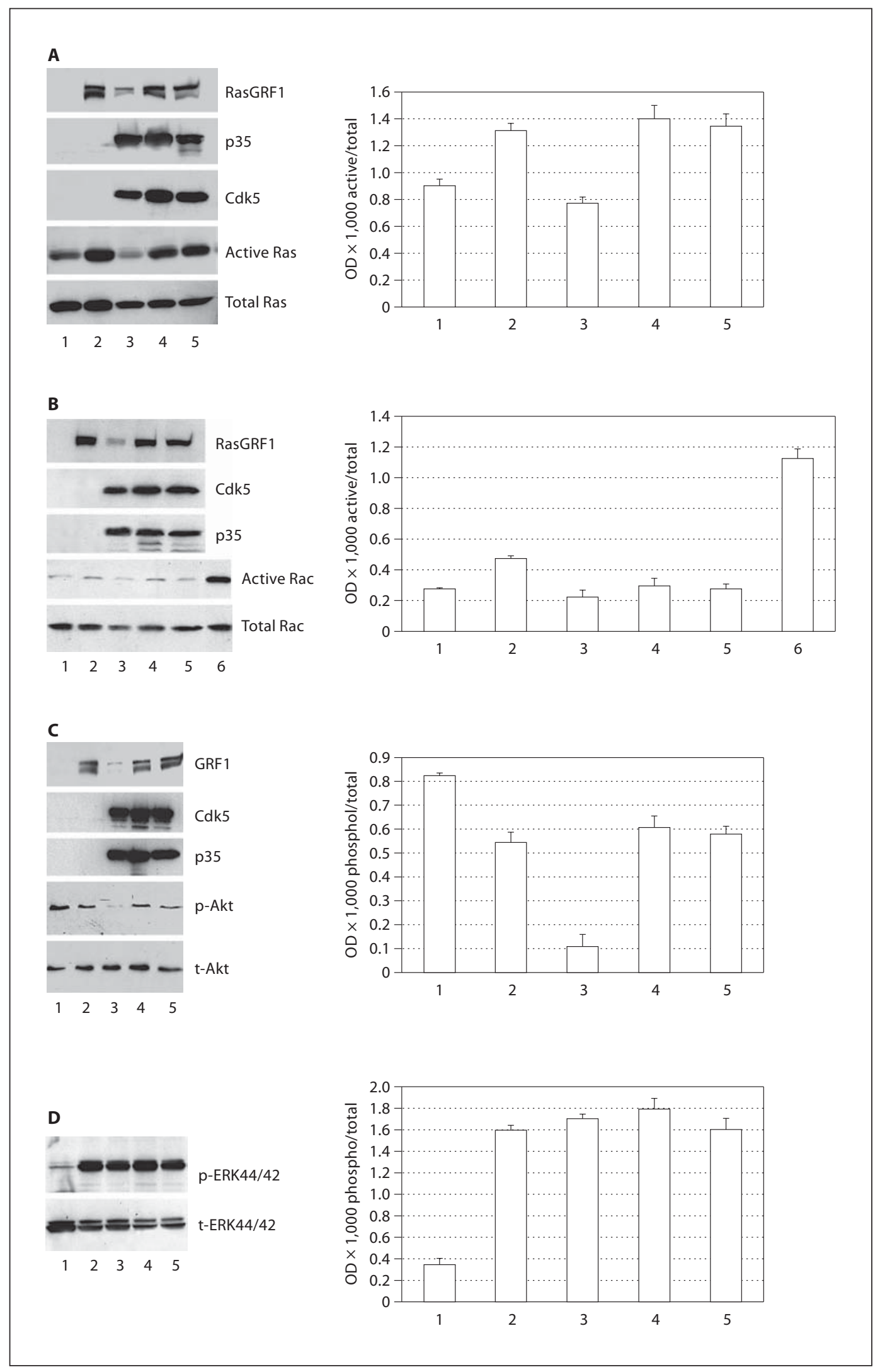


In our previous study with RasGRF2, we found that p35/Cdk5 phosphorylation caused a specific reduction of Rac but not Ras activity. Moreover, Erk1/2 activation was reduced leading to a modulation of MAP1b distribution but not that of tau, MAP2 or actin [21]. Thus, we wanted to determine whether $\mathrm{p} 35 / \mathrm{Cdk} 5$ phosphorylation of RasGRF1 caused similar specific downstream effects. We found that phosphorylation of RasGRF1 by p35/Cdk 5 did not significantly affect Rac activity (fig. 7B). The slight decrease in Rac activity with RasGRF1+p35+Cdk5 is insignificant when normalized to total Rac levels (fig. 7B, lane 3). Rac activity assays were tested by incubating empty vector transfected lysate with GTP before performing binding and pull-down assay and this exhibited highest levels of Rac activity as expected. However, phosphorylation of RasGRF1 by p35+Cdk 5 caused a reduction in phospho-Akt levels (fig. 7C, lane 3) compared to the transfection of empty vector (fig. 7C, lane 1) or with RasGRF1 alone (fig. 7C, lane 2). The transfection of RasGRF1 with p35+dominant-negative Cdk5 (fig. 7C, lane 4) and of RasGRF1 ${ }^{\text {Ser-Ala } 731}$ mutant with $\mathrm{p} 35 / \mathrm{Cdk} 5$ restored the levels of phospho-Akt to that found in the

Fig. 7. Cdk5 phosphorylation of RasGRF1 reduces Ras activity and subsequent Akt activation. A To detect active Ras, CHO cells were transfected with empty vector+Ras (1), RasGRF1+Ras (2), RasGRF1+p35+Cdk5+Ras (3), RasGRF1+p35+dominant-negative Cdk5+Ras (4), and RasGRF1mut731+p35+Cdk5+Ras (5). Cells were incubated with GST-c-Raf and were washed and then prepared for SDS-PAGE by boiling in $2 \times$ SDS-sample buffer. Samples were transferred onto nitrocellulose and subjected to Western blotting using anti-Ras antibody. Total Ras levels were immunodetected from lysates. B To detect active Rac, CHO cells were transfected with empty vector (1), RasGRF1 (2), RasGRF1+p35+Cdk5 (3), RasGRF1+p35+dominant-negative Cdk5 (4), RasGRF1mut731+p35+Cdk5 (5), and EV+GTP (6). Lysates were incubated with GST-PAK1-BD and samples were subjected to SDS-PAGE and Western blotting. Quantification of repeated Rac assays showed that any decrease in Rac activity was insignificant. C To determine the downstream effect of the modulation of RasGRF1-mediated Ras activity by p35/Cdk5, the activity of Akt was investigated. Lysates from $\mathrm{CHO}$ cells transfected with empty vector+Ras (1), RasGRF1+Ras (2), RasGRF1+p35+Cdk5+Ras (3), RasGRF1+p35+dominant-negative Cdk5+Ras (4), and RasGRF1mut731+p35+Cdk5+Ras (5) were subjected to SDS-PAGE and Western blotting for phosphoand total Akt levels. D To investigate whether p35/Cdk5 affects Erk1/2 activity, lysates in (C) were immunodetected for phospho and total Erk1/2. All data are representative Western blots of 3 separate experiments and quantification is shown in the bar graphs on the right.

p35/Cdk5 Phosphorylates RasGRF1 absence of p35/Cdk5. Surprisingly, Erk1/2 activity was unaffected when RasGRF1 was co-transfected with p35+Cdk5 (fig. 7D).

\section{Discussion}

RasGRF1 and RasGRF2 are guanine nucleotide exchange factors (GEFs) that display a high degree of homology to each other and both regulate the transition of inactive GDP-bound Ras and Rac to their active GTPbound forms. We previously showed that RasGRF2 was phosphorylated by $\mathrm{p} 35 / \mathrm{Cdk} 5$ on serine 737 [21]. The corresponding site in RasGRF1 is serine 731 and we found that $\mathrm{p} 35 / \mathrm{Cdk} 5$ associated with and phosphorylated RasGRF1 on this site. We consistently found that co-transfection of RasGRF1 with p35/Cdk5 in CHO cells resulted in a decrease in RasGRF1 steady state levels. The overexpression of either the dominant-negative Cdk5 or the phosphodeficient RasGRF1 mutant reversed this effect. Additionally, we found that in vivo levels of RasGRF1 in p35 knockout brain lysates were increased compared to their wild-type littermates. Cortical neurons derived from Cdk5 knockout embryos also contained higher, amounts of RasGRF1 compared to neurons from wildtype embryos. The overexpression of RasGRF1 and its reduction by overexpression of $\mathrm{p} 35 / \mathrm{Cdk} 5$ resulted in nuclear condensation in COS-7 cells and cortical neurons. When p35+Cdk5+RasGRF1+Ras were co-transfected into COS-7 cells, nuclei are compacted and this is also seen when RasGRF1 is overexpressed. All four proteins are normally expressed in neurons and perhaps the overexpression of all 4 proteins are required for normal function. However, levels of expression in this system may not accurately mimic a neuronal system and if one is higher, then the system is imbalanced. These data showed that RasGRF1 levels have to be tightly regulated for normal neuronal function and organization. A striking co-incidence was that Cdk5 knockout neurons start to exhibit condensation of nuclei and subsequent death after 3 days in culture. RasGRF1 expression is also apparent in neurons at 3 days in culture (data not shown). Thus, when RasGRF1 levels are deregulated through the absence of Cdk5 activity, neuronal nuclear condensation occurs.

RasGRF1 is also phosphorylated by protein kinase A [29] and by nonreceptor tyrosine kinase ACK1 [30] to modulate Ras activity, introducing the phenomenon of kinase-mediated regulation of RasGRF1 signaling. Previous reports have shown that the phosphorylation by PKA and ACK activate Ras and Rac without affecting 
RasGRF1 levels [30-32]. Thus, the phosphorylation of RasGRF1, by various kinases, has very different biological functions. RasGRF1 is localized to the plasma membrane through its pleckstrin homology $(\mathrm{PH})$ domain, although the exact mechanism of this is not fully understood but is thought to involve binding to membrane phospholipids or membrane-associated proteins such as G-protein $\beta \gamma$ subunits [33]. Interestingly, p35 is also localized to the plasma membrane through a myristoylation sequence found close to its amino-terminal [34]. Additionally, Ras is also localized to the inner leaflet of the plasma membrane which is important for its signaling role and brings it into close proximity with RasGRF1 and p35/Cdk5. Surprisingly, we found that p35/Cdk5 phosphorylation and subsequent reduction of RasGRF1 levels did not affect RasGRF1-mediated Erk1/2 activity contrary to reports from other groups $[35,36]$. We can only suggest that in the $\mathrm{CHO}$ cell system used in the experiments described, Erk1/2 activity was not controlled by RasGRF1. Indeed, in our previous studies, Erk1/2 activity in this system, was controlled by RasGRF2-mediated Rac activation.

In RasGRF1 knockout mice, ischemia-induced CREB activation is reduced and neuronal damage is enhanced [37]. CREB transcription factor is a survival-promoting factor. These findings are corroborated by our findings since in our experiments, the overexpression of p35/ Cdk 5 causes a decrease in RasGRF1 levels, a subsequent reduction in Ras activation, a reduction in active Akt (a marker of cell survival) linked to the disintegration of neuronal nuclei. Another report showed both RasGRF1 and RasGRF2 couple N-methyl-D-aspartate glutamate receptors to the activation of the Ras/Erk pathways [36]. Members of the N-methyl-D-aspartate class of glutamate receptors (NMDARs) are critical for development, synaptic transmission, learning and memory. NMDARs are phosphorylated by serine/threonine and tyrosine kinases to regulate its activity and Cdk 5 has been shown to phosphorylate the NR2A subunit of NMDA receptors [38].

Another paradigm where the interaction between RasGRF1 and Cdk5 activity may be of importance is in $\beta$ cells of the pancreas. RasGRF1 was originally thought to be expressed exclusively in the CNS; however, recent reports have shown that RasGRF1 is expressed and is required for $\beta$ cell development and glucose homeostasis [39]. RasGRF1 knockout mice exhibit reduced body weight, glucose intolerance and hypoinsulinemia due to a reduction in $\beta$ cells which are manifestations of preclinical type 2 diabetes [39]. Interestingly, p35/Cdk5 ex- pression and activity has also been found in these pancreatic $\beta$ cells induced by glucose [40]. These data introduce yet another possible role for $\mathrm{p} 35 / \mathrm{Cdk} 5$ and phosphorylation of RasGRF1 may not only be involved in neuronal function, but may also modulate RasGRF1 levels to influence $\beta$ cell development. This may contribute to normal pancreatic function or the development of type 2 diabetes, areas that require further study.

We propose that tight regulation of RasGRF1 levels is required for proper cellular organization. This hypothesis relies on a homeostasis between Cdk 5 activity levels and levels of critical signaling intermediates such as RasGRF1 for normal cell function. The tight regulation of RasGRF1 levels is inextricably linked to its control of Ras activation. It is clear that a deletion of RasGRF1 in mice is detrimental to long-term potentiation (LTP) with some dispute over which area in the brain is responsible for this $[41,42]$. The elevated levels of RasGRF1 in p35 and Cdk 5 knockout mice also seem to play a role in synaptic plasticity. The loss of Cdk 5 activity affects the phosphorylation of a number of synaptic proteins and has been shown to affect LTP $[38,43]$. Therefore, RasGRF1 levels and activity could be responsible for synaptic abnormalities in these paradigms. We also believe that regulation of Ras signaling mediated by RasGRF1 is regulated by local $\mathrm{Ca}^{2+}$ concentrations (fig. 8). Additionally, we believe that recognition of substrates by enzymes is crucial to function. $\mathrm{Ca}^{2+}$ is an intracellular signal which is responsible for vast array of cellular processes. An increase in intracellular calcium can be generated from sources inside as well as outside the cell. The endoplasmic reticulum network is an internal source where $\mathrm{Ca}^{2+}$ release is facilitated under the control of the channels. External $\mathrm{Ca}^{2+}$ enters the cell through voltage-operated channels, store-operated channels (upon depletion of internal $\mathrm{Ca}^{2+}$ levels) and receptor-operated channels that function upon ligand binding [reviewed in 44-46]. Together, the coordinated actions of these external and internal channels cause a complex increase of intracellular $\mathrm{Ca}^{2+}$. The coupling of the $\mathrm{Ca}^{2+}$ signal and the signal transduction responses seems to depend on the duration, amplitude and frequency of the local $\mathrm{Ca}^{2+}$ concentration at a given place in the cell, at a given time. Initial findings implicated $\mathrm{Ca}^{2+}$ influx in Ras activation which was proven upon discovery of $\mathrm{Ca}^{2+} /$ calmodulin activation of RasGRF1 [47-49]. Importantly, recent data showed that Cdk 5 activity is also regulated by $\mathrm{Ca}^{2+}$ levels [43]. A glutaminergic-mediated calcium influx causes a calmodulin-dependent activation of $\mathrm{Cdk} 5$ which autophosphorylates $\mathrm{p} 35$, which is then ubiquitinated and targeted for 


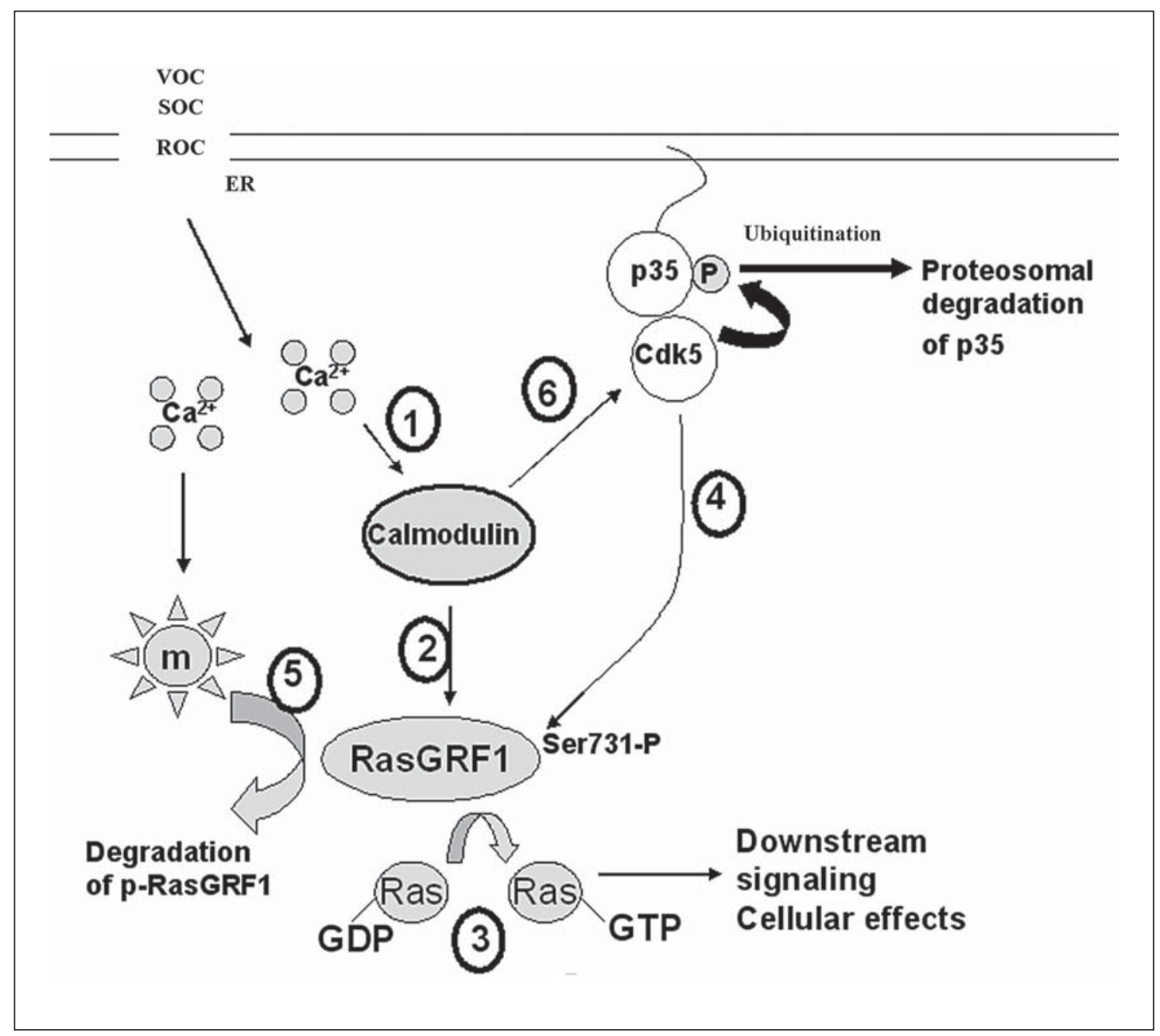

Fig. 8. Schematic diagram of ' $\mathrm{Ca}^{2+}$ sensing' controlling RasGRF1 and Cdk5 activities. Calcium enters the cell through a variety of sources such as receptor-operated (ROC), voltage-operated (VOC), store-operated (SOC) and internal stores such as endoplasmic reticulum (ER) and binds to calmodulin (1). $\mathrm{Ca}^{2+}$-calmodulin then binds to RasGRF1 to facilitate its activation (2). Upon activation, RasGRF1 facilitates the conversion of inactive Ras (Ras-GDP) to its active form (Ras-GTP) with downstream signaling and cellular effects (3). When Ras activation needs to be reduced, Cdk5, when associated with its activator p35, phosphorylates RasGRF1 on serine ${ }^{731}$ (4). The phosphorylated form is then recognized by m-calpain whose activity is also controlled by local $\mathrm{Ca}^{2+}$ concentrations (5). When Ras activation needs to be increased, $\mathrm{Ca}^{2+}$-calmodulin causes $\mathrm{p} 35$ phosphorylation (in response to a glutaminergic-mediated calcium influx) which then targets the activator for ubiquitination and subsequent degradation by the proteosome. This will increase RasGRF1 levels and Ras activation.

degradation by the proteosome [50]. This $\mathrm{Ca}^{2+}$-dependent process also relies on an enzyme's ability to recognize levels of phosphorylated proteins such as RasGRF1 and p35. We also believe that phosphorylated RasGRF1 levels cross a threshold and is then recognized by $\mathrm{m}$-calpain and degraded. Similarly, phosphorylated p35 crosses a threshold where an ubiquitin ligase attaches ubiqui- tin and then directs p35 to the proteasome for degradation. The tight regulation of $\mathrm{Ca}^{2+}$ levels which mediate RasGRF1 activation and Cdk5 activity, control levels of Ras activity which is critical for normal neuronal organization and function.

In this report, we have uncovered a novel pathway of regulation of RasGRF1 steady state levels through phos- 
phorylation by $\mathrm{p} 35 / \mathrm{Cdk} 5$. We have shown that levels of RasGRF1 are critical in neuronal nuclear organization evident by both overexpression and reduction of RasGRF1. This report, with our previous findings, places $\mathrm{Cdk} 5$ in a central regulatory role, where phosphorylation of two distinct GEFs have different biological effects in neurons. The phosphorylation of RasGRF2 modulated MAP1b distribution while that of RasGRF1 controlled nuclear organization in neurons. Further investigation to identify neuronal factors important for neuronal organization needs to be carried out and would provide information about fundamental processes involved in nuclear integrity. The importance of the regulatory role Cdk5 plays in nervous system signal transduction cascades has been demonstrated and will be critical to our understanding in these processes.

\section{References}

1 Tsai LH, Delalle I, Caviness VS Jr, Chae T, Harlow E: p35 is a neural-specific regulatory subunit of cyclin-dependent kinase 5 . Nature 1994;371:419-423.

-2 Humbert S, Lanier LM, Tsai LH: Synaptic localization of p39, a neuronal activator of cdk5. Neuroreport 2000;11:2213-2216.

- 3 Nikolic M, Dudek H, Kwon YT, Ramos YF, Tsai LH: The cdk5/p35 kinase is essential for neurite outgrowth during neuronal differentiation. Genes Dev 1996;10:816-825.

4 Ohshima T, Ward JM, Huh CG, et al: Targeted disruption of the cyclin-dependent kinase 5 gene results in abnormal corticogenesis, neuronal pathology and perinatal death. Proc Natl Acad Sci USA 1996;93:1117311178.

5 Kesavapany S, Lau KF, McLoughlin DM, et al: p35/cdk5 binds and phosphorylates betacatenin and regulates beta-catenin/presenilin-1 interaction. Eur J Neurosci 2001;13: 241-247.

-6 Kwon YT, Gupta A, Zhou Y, Nikolic M, Tsai LH: Regulation of N-cadherin-mediated adhesion by the p35-Cdk5 kinase. Curr Biol 2000;10:363-372.

-7 Shuang R, Zhang L, Fletcher A, Groblewski GE, Pevsner J, Stuenkel EL: Regulation of Munc-18/syntaxin 1A interaction by cyclindependent kinase 5 in nerve endings. J Biol Chem 1998;273:4957-4966.

-8 Rosales JL, Nodwell MJ, Johnston RN, Lee KY: Cdk5/p25(nck5a) interaction with synaptic proteins in bovine brain. J Cell Biochem 2000;78:151-159.

-9 Fletcher AI, Shuang R, Giovannucci DR, Zhang L, Bittner MA, Stuenkel EL: Regulation of exocytosis by cyclin-dependent kinase 5 via phosphorylation of Munc18. J Biol Chem 1999;274:4027-4035.

- 10 Matsubara M, Kusubata M, Ishiguro K, Uchida T, Titani K, Taniguchi H: Site-specific phosphorylation of synapsin I by mitogen-activated protein kinase and Cdk5 and its effects on physiological functions. J Biol Chem 1996;271:21108-21113.
11 Tomizawa K, Cai XH, Moriwaki A, Matsushita M, Matsui H: Involvement of cyclin-dependent kinase 5/p35(nck5a) in the synaptic reorganization of rat hippocampus during kindling progression. Jpn J Physiol 2000;50: 525-32.

12 Tan TC, Valova VA, Malladi CS, et al: Cdk5 is essential for synaptic vesicle endocytosis. Nat Cell Biol 2003;5:701-710.

13 Bibb JA, Snyder GL, Nishi A, et al: Phosphorylation of DARPP-32 by Cdk 5 modulates dopamine signalling in neurons. Nature 1999; 402:669-671.

14 Takahashi S, Ohshima T, Cho A, et al: Increased activity of cyclin-dependent kinase 5 leads to attenuation of cocaine-mediated dopamine signaling. Proc Natl Acad Sci USA 2005;102:1737-1742.

15 Fu AK, Fu WY, Cheung J, et al: Cdk5 is involved in neuregulin-induced AChR expression at the neuromuscular junction. Nat Neurosci 2001;4:374-381.

16 Bibb JA, Chen J, Taylor JR, et al: Effects of chronic exposure to cocaine are regulated by the neuronal protein Cdk5. Nature 2001;410: 376-380.

17 Fu AK, Ip FC, Fu WY, et al: Aberrant motor axon projection, acetylcholine receptor clustering, and neurotransmission in cyclin-dependent kinase 5 null mice. Proc Natl Acad Sci USA 2005; 102:15224-15229.

18 Sharma P, Veeranna, Sharma M, et al: Phosphorylation of MEK1 by cdk5/p35 downregulates the mitogen-activated protein $\mathrm{ki}$ nase pathway. J Biol Chem 2002;277: 528-534.

19 Zhen X, Goswami S, Abdali SA, Gil M, Bakshi K, Friedman E: Regulation of cdk5 and CaMK II by PI-linked dopamine receptor in rat brain. Mol Pharmacol 2004.

20 Li BS, Zhang L, Takahashi S, et al: Cyclindependent kinase 5 prevents neuronal apoptosis by negative regulation of c-Jun N-terminal kinase 3. EMBO J 2002;21:324-333.
21 Kesavapany S, Amin N, Zheng YL, et al:p35/ cyclin-dependent kinase 5 phosphorylation of ras guanine nucleotide releasing factor 2 (RasGRF2) mediates Rac-dependent extracellular signal-regulated kinase $1 / 2$ activity, altering RasGRF2 and microtubule-associated protein $1 b$ distribution in neurons. J Neurosci 2004;24:4421-4431.

22 Patzke H, Tsai LH: Calpain-mediated cleavage of the cyclin-dependent kinase-5 activator p39 to p29. J Biol Chem 2002;277:80548060 .

23 Lee MS, Kwon YT, Li M, Peng J, Friedlander RM, Tsai LH: Neurotoxicity induces cleavage of p35 to p25 by calpain. Nature 2000; 405:360-364.

24 Zheng YL, Kesavapany S, Gravell M, et al: A Cdk5 inhibitory peptide reduces tau hyperphosphorylation and apoptosis in neurons. EMBO J 2005;24:209-220.

25 Kesavapany S, Lau KF, Ackerley S, et al: Identification of a novel, membrane-associated neuronal kinase, cyclin-dependent kinase 5/ p35-regulated kinase. J Neurosci 2003;23: 4975-4983.

26 Kesavapany S, Banner SJ, Lau KF, et al: Expression of the Fe65 adapter protein in adult and developing mouse brain. Neuroscience 2002;115:951-960.

27 Amin ND, Albers W, Pant HC: Cyclin-dependent kinase $5(\mathrm{cdk} 5)$ activation requires interaction with three domains of p35. J Neurosci Res 2002;67:354-362.

28 Dhavan R, Greer PL, Morabito MA, Orlando LR, Tsai LH: The cyclin-dependent kinase 5 activators p35 and p39 interact with the alpha-subunit of $\mathrm{Ca}^{2+} /$ calmodulin-dependent protein kinase II and alpha-actinin-1 in a calcium-dependent manner. J Neurosci 2002;22:7879-7991.

29 Baouz S, Jacquet E, Accorsi K, et al: Sites of phosphorylation by protein kinase $\mathrm{A}$ in CDC25Mm/GRF1, a guanine nucleotide exchange factor for Ras. J Biol Chem 2001;276: 1742-1729. 
-30 Kiyono M, Kato J, Kataoka T, Kaziro Y, Satoh T: Stimulation of Ras guanine nucleotide exchange activity of Ras-GRF1/CDC25(Mm) upon tyrosine phosphorylation by the Cdc42-regulated kinase ACK1. J Biol Chem 2000;275:29788-29793.

>31 Kiyono M, Kaziro Y, Satoh T: Induction of rac-guanine nucleotide exchange activity of Ras-GRF1/CDC25(Mm) following phosphorylation by the nonreceptor tyrosine kinase Src. J Biol Chem 2000;275:5441-5446.

-32 Yang H, Cooley D, Legakis JE, Ge Q, Andrade R, Mattingly RR: Phosphorylation of the Ras-GRF1 exchange factor at Ser916/898 reveals activation of Ras signaling in the cerebral cortex. J Biol Chem 2003;278:1327813285.

-33 Buchsbaum R, Telliez JB, Goonesekera S, Feig LA: The N-terminal pleckstrin, coiledcoil, and IQ domains of the exchange factor Ras-GRF act cooperatively to facilitate activation by calcium. Mol Cell Biol 1996;16: 4888-4896.

-34 Patrick GN, Zukerberg L, Nikolic M, de la Monte S, Dikkes P, Tsai LH: Conversion of p35 to p25 deregulates Cdk5 activity and promotes neurodegeneration. Nature 1999; 402:615-622.

>35 Krapivinsky G, Krapivinsky L, Manasian Y, et al: The NMDA receptor is coupled to the ERK pathway by a direct interaction between NR2B and RasGRF1. Neuron 2003;40:775784 .
36 Tian X, Gotoh T, Tsuji K, Lo EH, Huang S, Feig LA: Developmentally regulated role for Ras-GRFs in coupling NMDA glutamate receptors to Ras, Erk and CREB. EMBO J 2004; 23:1567-1575.

>37 Tian X, Feig LA: Basis for signaling specificity difference between Sos and Ras-GRF guanine nucleotide exchange factors. J Biol Chem 2001;276:47248-47256.

38 Li BS, Sun MK, Zhang L, et al: Regulation of NMDA receptors by cyclin-dependent kinase-5. Proc Natl Acad Sci USA 2001;98: 12742-12747.

39 Font de Mora J, Esteban LM, Burks DJ, et al: Ras-GRF1 signaling is required for normal beta-cell development and glucose homeostasis. EMBO J 2003;22:3039-3049.

40 Ubeda M, Kemp DM, Habener JF: Glucoseinduced expression of the cyclin-dependent protein kinase 5 activator p35 involved in Alzheimer's disease regulates insulin gene transcription in pancreatic beta-cells. Endocrinology 2004;145:3023-3031.

41 Giese KP, Friedman E, Telliez JB, et al: Hippocampus-dependent learning and memory is impaired in mice lacking the Ras-guaninenucleotide releasing factor 1 (Ras-GRF1). Neuropharmacology 2001;41:791-800

42 Brambilla R, Gnesutta N, Minichiello L, et al: A role for the Ras signalling pathway in synaptic transmission and long-term memory. Nature 1997;390:281-286.

43 Wei FY, Tomizawa K, Ohshima T, et al: Control of cyclin-dependent kinase $5(\mathrm{Cdk} 5)$ activity by glutamatergic regulation of p35 stability. J Neurochem 2005;93:502-512.
44 Cullen PJ, Lockyer PJ: Integration of calcium and Ras signalling. Nat Rev Mol Cell Biol 2002;3:339-48.

45 Walker SA, Lockyer PJ, Cullen PJ: The Ras binary switch: an ideal processor for decoding complex $\mathrm{Ca}^{2+}$ signals? Biochem Soc Trans 2003;31:966-969.

46 Walker SA, Cullen PJ, Taylor JA, Lockyer PJ: Control of Ras cycling by $\mathrm{Ca}^{2+}$. FEBS Lett 2003;546:6-10.

47 Shou C, Farnsworth CL, Neel BG, Feig LA: Molecular cloning of cDNAs encoding a guanine-nucleotide-releasing factor for Ras p21. Nature 1992;358:351-354.

48 Farnsworth CL, Freshney NW, Rosen LB, Ghosh A, Greenberg ME, Feig LA: Calcium activation of Ras mediated by neuronal exchange factor Ras-GRF. Nature 1995;376: 524-527.

49 Martegani E, Vanoni M, Zippel R, et al: Cloning by functional complementation of a mouse cDNA encoding a homologue of CDC25, a Saccharomyces cerevisiae RAS activator. Embo J 1992;11:2151-2157.

50 Patrick GN, Zhou P, Kwon YT, Howley PM, Tsai LH: p35, the neuronal-specific activator of cyclin-dependent kinase $5(\mathrm{Cdk} 5)$ is degraded by the ubiquitin-proteasome pathway. J Biol Chem 1998;273:24057-24064. 